\title{
Comparison of Sleep and Respiratory Parameters of Obstructive Sleep Apnea Patients during Diagnostic and 2 Hours Automatic Positive Airway Pressure Split-night Titration: A Descriptive Study
}

\author{
Arup K Halder ${ }^{1} \odot$, Arpita C Halder ${ }^{2}$
}

\begin{abstract}
Aims and objectives: Obstructive sleep apnea (OSA) is a common disorder manifested with snoring, daytime sleepiness, fatigue, metabolic, and cardiovascular symptoms. Manual continuous positive airway pressure (CPAP) titration is the gold standard to determine the amount of positive pressure required to abolish the airflow limitations. The current American Academy of Sleep Medicine (AASM) criteria for manual titration are very stringent, elegant but difficult. The AASM protocol does not favor the use of automatic positive airway pressure (APAP) in a split-night study. This study was done to look into changes in sleep and respiratory parameters following diagnostic polysomnography (PSG) and subsequent APAP titration, as a split-night protocol.

Materials and method: Records of 80 patients were scrutinized who had done level 1 PSG in a sleep laboratory in Kolkata, India. The laboratory used APAP titration for all kinds of titrations. This is a descriptive study, where data were compared between diagnostic and therapeutic nights of the same patients, done as a split-night study.

Results: The diagnostic night was denoted by visit 1 and therapeutic night as visit 2; the study was done as a split-night study. The rapid eye movement (REM) sleep time was found to be significantly increased from 15.08 minutes in V1 (SD 16.26) to 29.69 minutes (24.45) in V2 with a $p<0.001$. The total respiratory events were found to be significantly reduced from baseline median value of 206-14 in the follow-up visit posttreatment, $p<0.001$ as computed by Wilcoxon's signed-rank test. The REM SpO $\mathrm{S}_{2}$ was found to be significantly increased from baseline value of $90.87 \pm 7.105$ to $93.29 \pm 6.312$ in the follow-up visit posttreatment, $p<0.001$ as computed by paired sample $t$-test.

Discussion and conclusion: The wake stages, N1 and N2 sleep, were reduced significantly in the therapeutic night than diagnostic night, but N3 sleep was increased in therapeutic night, though statistically not significant. The total arousals and arousal index were also decreased significantly, although there were wide interindividual variations. So overall, patients had an improved sleep architecture during therapeutic night and often with REM rebound. Overall respiratory parameters showed very significant improvement in terms of apnea and hypopnea index (AHI). Oximetry data showed very significant improvements in terms of oxygen saturation, nadir oxygen saturation, and REM time oxygen level. So we can formulate the hypothesis that even a 2 hours split-night APAP titration can perform a good titration and significant improvements in sleep and respiratory parameters. Keywords: Automatic positive airway pressure titration, Continuous positive airway pressure titration, Obstructive sleep apnea, Polysomnography, Split-night study.

Indian Journal of Sleep Medicine (2021): 10.5005/jp-journals-10069-0076
\end{abstract}

\section{INTRODUCTION}

Obstructive sleep apnea (OSA) is a common disorder manifested with snoring, daytime sleepiness, fatigue, metabolic, and cardiovascular symptoms. ${ }^{1}$ It is diagnosed with overnight polysomnography (PSG), either as a full-night or split-night study protocol. ${ }^{2-6}$ The treatment of OSA is positive airway pressure (PAP) therapy, and PSG with manual continuous PAP (CPAP) titration is the gold standard to determine the amount of positive pressure required to abolish the airflow limitations. ${ }^{2,7}$ The current American Academy of Sleep Medicine (AASM) criteria for manual titration are very stringent, elegant but difficult. It requires a competent technician, who should be competently conversed to scoring also. But there is a paucity of such trained technicians in various parts of the world. It often leads to automatic PAP titration, known as APAP titration. ${ }^{8,9}$ The AASM protocol does not favor the use of APAP in the split-night study. Current APAP titration guideline recommends at least three nights titrations for getting an assessment of pressure required to abolish airflow limitations and thus obtaining optimal manual CPAP pressure to be prescribed thereafter. ${ }^{10-14}$ This study was done to look into changes in sleep and respiratory parameters following diagnostic PSG and subsequent APAP titration done same night, like split-night protocol.
1Department of Pulmonology and Sleep, Institute of Sleep Sciences, Kolkata, West Bengal, India

${ }^{2}$ Department of Anatomy, College of Medicine and Sagore Dutta Hospital, Kolkata, West Bengal, India

Corresponding Author: Arup K Halder, Department of Pulmonology and Sleep, Institute of Sleep Sciences, Kolkata, West Bengal, India, e-mail: haldar.arup@yahoo.com

How to cite this article: Halder AK, Halder AC. Comparison of Sleep and Respiratory Parameters of Obstructive Sleep Apnea Patients during Diagnostic and 2 Hours Automatic Positive Airway Pressure Split-night Titration: A Descriptive Study. Indian J Sleep Med 2021;16(3):77-81.

Source of support: Nil

Conflict of interest: None

\section{Materials and Methods}

Records of 80 patients were scrutinized who had done level 1 PSG in a sleep laboratory in Kolkata, India. All of these patients were referred for level 1 PSG with a request for split-night titration if required. The split-night study was done for moderate-

(O) The Author(s). 2021 Open Access This article is distributed under the terms of the Creative Commons Attribution 4.0 International License (https://creativecommons. org/licenses/by-nc/4.0/), which permits unrestricted use, distribution, and non-commercial reproduction in any medium, provided you give appropriate credit to the original author(s) and the source, provide a link to the Creative Commons license, and indicate if changes were made. The Creative Commons Public Domain Dedication waiver (http://creativecommons.org/publicdomain/zero/1.0/) applies to the data made available in this article, unless otherwise stated. 
to-severe OSA patients or OSA patients with significant oxygen desaturations. The laboratory used APAP titration for all kinds of titrations. The patients first had undergone a diagnostic night for 4 hours with the following channels: electroencephalograms (EEG), electrooculograms (EOG), electromyograms (EMG), electrocardiograms (ECG), nasal pressure transducer, oral thermistor, thoracic and abdominal plethysmographic belts, pulse oximeter, and position and snore sensors. The APAP titration was carried out with similar channels except for the flow channels of nasal pressure transducers and oral thermistors. The titrations were done mainly with nasal interface mainly with full face masks in few patients. The choices of masks were done during preprocedure counseling. The data were manually validated by the trained doctor according to AASM protocol. Data of those patients were analyzed, who completed the study and for whom most of the channel data were available.

This is a descriptive study where data were compared between diagnostic and therapeutic nights of the same patients. Suitable statistical analysis was done for the paired data with statistical software.

\section{Results}

\section{Statistical Methods}

Descriptive statistical analysis has been carried out in the present study. Results on continuous measurements are presented on mean $\pm S D$, and results on categorical measurements are presented in number (\%). The significance is assessed at a level of $5 \%$. The normality of data was tested by simultaneous Anderson-Darling test, Shapiro-Wilk test, and graphically by QQ plot. Analysis of variance (ANOVA) with post hoc Dunnett's test for adjustment method has been used to find the significance of study parameters between three or more groups of subjects. Paired $t$-test or Wilcoxon's signed-rank test was applied to detect any significant change of study parameters measured on two occasions for the same group of patients. A $p$ value of $<0.05$ was considered statistically significant. The statistical software, namely statistical analysis system (SAS) version 9.2 for Windows, SAS Institute Inc. Cary, North Carolina, United States and statistical package for social sciences (SPSS Complex Samples) version 21.0 for Windows, SPSS, Inc., Chicago, Illinois, United States, were used for the analysis of the data. Microsoft word 2010 and Microsoft Excel 2010 (Microsoft Corp, Redmond, Washington, United States) have been used to generate graphs and Tables 1 to 4 .

The paired data were obtained from a split-night study conducted on the same night, first as diagnostic night and followed by therapeutic night in a sample size of 80 . The diagnostic night was denoted by visit 1 and therapeutic night as visit 2 .

The total time in bed (TBD) was greater during diagnostic night than in therapeutic night, and it was 233.45 minutes (SD 43.66) during diagnostic night and 146.78 minutes (SD 35.62), with a $p<0.001$. The total sleep time (TST) was also significantly lower from diagnostic to therapeutic night from 205.71 minutes (SD 40.69) to 132.20 minutes (SD 28.21) with $p<0.001$, by paired $t$-test.

The non-REM (NREM) sleep time was found to be significantly reduced from 192.46 minutes (SD 39.86) to 112.76 minutes (SD 33.49) with a $p<0.001$ as computed by paired $t$-test. The REM sleep time was found to be significantly increased from 15.08 minutes (SD 16.26) to 29.69 minutes (24.45) with a $p<0.001$. The sleep time was found to be significantly increased from 8.44 minutes (SD 16.14) to 47.83 minutes (SD 40.68) with a $p<0.001$.
The wake after sleep onset (WASO) was found to be significantly decreased from 30.90 minutes (SD 24.91) to 14.88 minutes (SD 17.57), with $p<0.001$. The wake stage was significantly reduced from $13.69 \%$ (SD 11.14) to $9.49 \%$ (SD 9.58), with $p<0.002$. Stage N1 was significantly decreased from $13.25 \%$ (SD 7.62) to $9.53 \%$ (SD 9.46), with $p<0.002$. The stage N2 was also significantly decreased from $69.70 \%$ (SD 11.75) to $62.51 \%$ (SD 20.15), with $p<0.003$. But though N3 sleep increased from $11.95 \%$ (SD 7.55) to $13.34 \%$ (SD 11.47), it was not statistically significant with $p>0.307$. REM stage significantly increased from $5.20 \%$ (SD 6.31) to $14.66 \%$ (SD 17.42), with $p<0.001$. The total number of arousals was decreased from mean 121.16 (SD 80.60) to 42.91 (SD 39.13), with $p<0.001$. So, arousal index was also decreased significantly from mean 35.58 (SD 21.64) to 19.25 (16.70) with $p<0.001$.

\section{Inference}

The total respiratory events were found to be significantly reduced from baseline median value of 206-14 in the follow-up visit posttreatment, $p<0.001$ as computed by Wilcoxon's signed-rank test. The events of apnea were found to be significantly reduced from baseline median value of 121-6 in the follow-up visit posttreatment, $p<0.001$ as computed by Wilcoxon's signed-rank test.

The events of hypopnea were found to be significantly reduced from baseline median value of 36.5-6 in the follow-up visit posttreatment, $p<0.001$ as computed by Wilcoxon's signedrank test. The apnea and hypopnea index (AHI) was found to be significantly reduced from baseline median value of $60.5-6$ in the follow-up visit posttreatment, $p<0.001$ as computed by Wilcoxon's signed-rank test.

\section{Inference}

The $\mathrm{SpO}_{2}$ was found to be significantly increased from baseline value of $92.72 \pm 5.103$ to $94.77 \pm 3.692$ in the follow-up visit posttreatment, $p<0.001$ as computed by paired sample $t$-test. The nadir $\mathrm{SpO}_{2}$ was found to be significantly increased from baseline value of $70.14 \pm 16.172$ to $83.44 \pm 10.108$ in the follow-up visit posttreatment, $p<0.001$ as computed by paired sample $t$-test.

The REM $\mathrm{SpO}_{2}$ was found to be significantly increased from baseline value of $90.87 \pm 7.105$ to $93.29 \pm 6.312$ in the follow-up visit posttreatment, $p<0.001$ as computed by paired sample $t$-test.

\section{Discussion}

The TBD was more in diagnostic night than therapeutic night as patients were allowed almost 4 hours of diagnostic and 3 hours of therapeutic night time. TST also changed accordingly. NREM sleep time also changed in the same manner. But REM sleep time significantly increased during titration night, though there was a wide variation of REM sleep distribution between individuals. There may be two reasons for these-first, as therapeutic nights were mainly the early morning periods, the REM intensity was naturally high and secondly, correction of flow limitations resulted in REM rebound. The sleep time had very high interindividual variations but significantly increased on therapeutic night. The reason may be patient required an adjustment time with APAP before sleep. Interestingly, WASO significantly decreased in therapeutic night, although the data seem to vary widely among individuals. The wake stages, N1 and N2 sleep, were reduced significantly in therapeutic night than diagnostic night, but N3 sleep was increased in therapeutic night, though statistically not significant. The total arousals 
The Present Study Demonstrates the Utility of APAP Split-night Titration

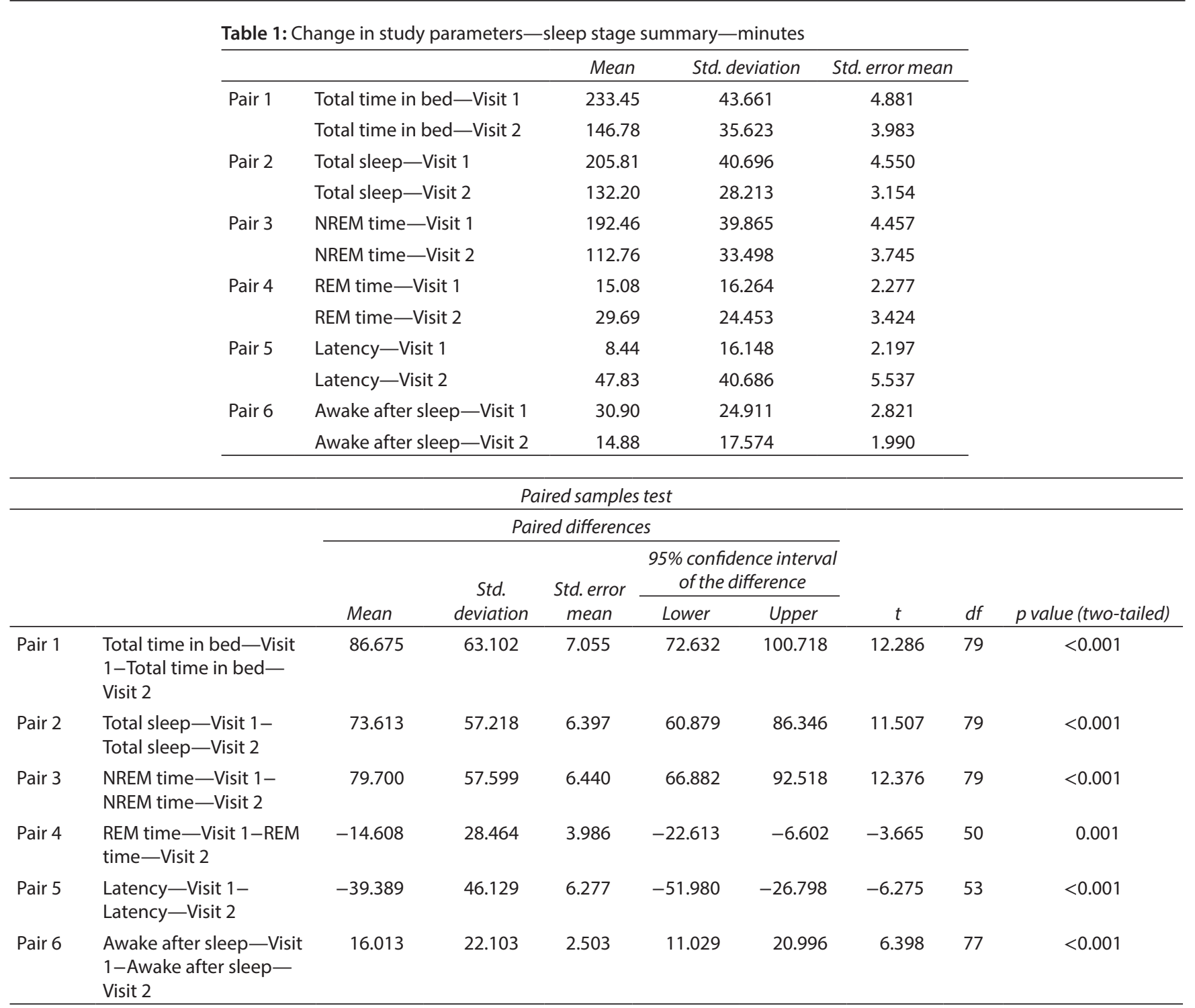

Table 2: Change in study parameters—sleep architecture-\%

\begin{tabular}{llcccc}
\hline \multicolumn{5}{c}{ Paired samples statistics } \\
\hline \multirow{2}{*}{ Pair 1 } & Nwake-Visit 1 & 80 & 13.69 & 11.114 & 1.447 \\
& Awake-Visit 2 & 80 & 9.49 & 9.580 & 1.247 \\
Pair 2 & N1-Visit 1 & 80 & 13.25 & 7.625 & 0.852 \\
& N1-Visit 2 & 80 & 9.53 & 9.463 & 1.058 \\
Pair 3 & N2-Visit 1 & 80 & 69.70 & 11.753 & 1.314 \\
& N2-Visit 2 & 80 & 62.51 & 20.158 & 2.254 \\
Pair 4 & N3-Visit 1 & 80 & 11.95 & 7.557 & 0.845 \\
& N3-Visit 2 & 80 & 13.34 & 11.473 & 1.283 \\
Pair 5 & REM-Visit 1 & 80 & 5.20 & 6.319 & 0.707 \\
& REM-Visit 2 & 80 & 14.66 & 17.421 & 1.948 \\
Pair 6 & Total arousals-Visit 1 & 80 & 121.16 & 80.602 & 9.012 \\
& Total arousals—Visit 2 & 80 & 42.91 & 39.136 & 4.376 \\
Pair 7 & Arousal index-Visit 1 & 80 & 35.58 & 21.644 & 2.420 \\
& Arousal index-Visit 2 & 80 & 19.25 & 16.706 & 1.868 \\
\hline & & & & & (Contd...)
\end{tabular}




\begin{tabular}{|c|c|c|c|c|c|c|c|c|c|}
\hline \multicolumn{10}{|c|}{ Paired samples test } \\
\hline & & \multicolumn{5}{|c|}{ Paired differences } & \multirow[b]{3}{*}{$t$} & \multirow[b]{3}{*}{$d f$} & \multirow[b]{3}{*}{$p$ (two-tailed } \\
\hline & & \multirow[b]{2}{*}{ Mean } & \multirow[b]{2}{*}{ Std. deviation } & \multirow{2}{*}{$\begin{array}{l}\text { Std. error } \\
\text { mean }\end{array}$} & \multicolumn{2}{|c|}{$\begin{array}{c}\text { 95\% confidence interval of } \\
\text { the difference }\end{array}$} & & & \\
\hline & & & & & Lower & Upper & & & \\
\hline Pair 1 & $\begin{array}{l}\text { Awake-Visit 1- } \\
\text { Awake-Visit } 2\end{array}$ & 4.203 & 10.177 & 1.325 & 1.551 & 6.856 & 3.172 & 58 & 0.002 \\
\hline Pair 2 & N1-Visit 1-N1-Visit 2 & 3.725 & 10.460 & 1.169 & 1.397 & 6.053 & 3.185 & 79 & 0.002 \\
\hline Pair 3 & N2-Visit 1-N2-Visit 2 & 7.188 & 20.604 & 2.304 & 2.602 & 11.773 & 3.120 & 79 & 0.003 \\
\hline Pair 4 & N3-Visit 1-N3-Visit 2 & -1.388 & 12.075 & 1.350 & -4.075 & 1.300 & -1.028 & 79 & 0.307 \\
\hline Pair 5 & $\begin{array}{l}\text { REM-Visit 1-REM- } \\
\text { Visit } 2\end{array}$ & -9.462 & 16.528 & 1.848 & -13.141 & -5.784 & -5.121 & 79 & $<0.001$ \\
\hline Pair 6 & $\begin{array}{l}\text { Total arousals-Visit 1- } \\
\text { Total arousals—Visit } 2\end{array}$ & 78.250 & 77.201 & 8.631 & 61.070 & 95.430 & 9.066 & 79 & $<0.001$ \\
\hline Pair 7 & $\begin{array}{l}\text { Arousal index-Visit 1- } \\
\text { Arousal index-Visit } 2\end{array}$ & 16.325 & 20.305 & 2.270 & 11.806 & 20.844 & 7.191 & 79 & $<0.001$ \\
\hline
\end{tabular}

Table 3: Changes in respiratory events

\begin{tabular}{|c|c|c|c|c|c|c|c|c|c|c|}
\hline & $N$ & Mean & Std.deviation & Minimum & Maximum & Median & Lower quartile & Upper quartile & Zvalue & $p$ value \\
\hline $\begin{array}{l}\text { Total Resp } \\
\text { events_Visit } 1\end{array}$ & 80 & 197.89 & 116.926 & 9 & 443 & 206.00 & 85.25 & 295.75 & -7.30 & $<0.001$ \\
\hline $\begin{array}{l}\text { Total Resp } \\
\text { events—Visit } 2\end{array}$ & 80 & 27.68 & 43.677 & 0 & 315 & 14.00 & 9.00 & 31.50 & & \\
\hline Apneas-Visit 1 & 80 & 145.68 & 111.260 & 2 & 418 & 121.00 & 44.25 & 235.00 & -7.11 & $<0.001$ \\
\hline Apneas-Visit 2 & 80 & 17.66 & 38.379 & 0 & 283 & 6.00 & 2.00 & 18.00 & & \\
\hline $\begin{array}{l}\text { Hypopneas- } \\
\text { Visit } 1\end{array}$ & 80 & 51.81 & 41.892 & 5 & 223 & 36.50 & 22.50 & 68.75 & -7.55 & $<0.001$ \\
\hline $\begin{array}{l}\text { Hypopneas- } \\
\text { Visit } 2\end{array}$ & 80 & 10.01 & 13.225 & 0 & 93 & 6.00 & 2.25 & 13.75 & & \\
\hline AHI—Visit 1 & 80 & 55.80 & 30.773 & 4 & 129 & 60.50 & 26.00 & 80.00 & -7.25 & $<0.001$ \\
\hline AHI—Visit 2 & 80 & 12.23 & 15.414 & 0 & 85 & 6.00 & 3.25 & 13.75 & & \\
\hline RDI—Visit 1 & 80 & 56.38 & 30.678 & 4 & 129 & 63.00 & 26.25 & 80.00 & -7.26 & $<0.001$ \\
\hline RDI-Visit 2 & 80 & 12.23 & 15.414 & 0 & 85 & 6.00 & 3.25 & 13.75 & & \\
\hline
\end{tabular}

and arousal index were also decreased significantly, although there were wide interindividual variations. So overall, patients had improved sleep architecture during therapeutic night and often with REM rebound.

Overall respiratory parameters showed very significant improvement in terms of $\mathrm{AHI}$, apneas and hypopneas. Oximetry data showed very significant improvements in terms of oxygen saturation, nadir oxygen saturation, and REM time oxygen level. As per AASM, a good titration reduces respiratory disturbance index (RDI) $\leq 10$ or by $50 \%$ if the baseline RDI $<15$ and should include supine REM sleep that is not continually interrupted by spontaneous arousals or awakenings at the selected pressure. ${ }^{15}$ The current result is consistent with good titration, even during APAP split night.

Still now there is a paucity of data on head-to-head comparison between manual CPAP titration and APAP titration, especially done as a split-night protocol. One study done in this field from Egypt showed that the use of APAP was equal to manual titration in this group of patients and required lesser time to reach the therapeutic pressure. ${ }^{16}$ Such a benefit may translate to earlier commencement of treatment for many patients.
The weakness of this study was a small sample size and no direct control group. The study also has not assessed whether this quick fixing translates to improved patient adherence to PAP devices. A large-scale multicenter randomized controlled trial (RCT) with proper control groups can probably answer this issue in a better fashion.

\section{Conclusion and Hypothesis Formulation}

So we can draw the following conclusion from the study:

- Even a 2 hours APAP titration results in a significant improvement of sleep architecture in OSA patients.

- Even a 2 hours APAP titration results in a very significant improvement of airflow limitations and oxygenation in OSA patients.

So we can formulate the hypothesis that even a 2 hours splitnight APAP titration can perform a good titration and significant improvements in sleep and respiratory parameters. Whether it can be recommended as a split-night titration method will require a RCT and comparison with the gold standard CPAP titration. 
Table 4: Changes in oxygen saturation

\begin{tabular}{llllccc}
\hline \multicolumn{7}{c}{ Paired samples statistics-oxygen saturation } \\
\hline Oxygen saturation & Mean & $N$ & Std. deviation & Std. error mean & $p$ \\
\hline Pair 1 & Awake-Visit 1 & 92.72 & 74 & 5.103 & 0.593 & $<0.001$ \\
& Awake-Visit 2 & 94.77 & 74 & 3.692 & 0.429 & \\
Pair 2 & Nadir SpO 2 -Visit 1 & 70.14 & 79 & 16.172 & 1.819 & $<0.001$ \\
& Nadir SpO 2 -Visit 2 & 83.44 & 79 & 10.108 & 1.137 & \\
Pair 3 & REM-Visit 1 & 90.87 & 45 & 7.105 & 1.059 & 0.035 \\
& REM-Visit 2 & 93.29 & 45 & 6.312 & 0.941 & \\
\hline
\end{tabular}

\begin{tabular}{|c|c|c|c|c|c|c|c|c|c|}
\hline \multicolumn{10}{|c|}{ Paired samples test } \\
\hline & & \multicolumn{5}{|c|}{ Paired differences } & \multirow[b]{3}{*}{ tvalue } & \multirow[b]{3}{*}{$d f$} & \multirow[b]{3}{*}{$p$ (two-tailed) } \\
\hline & & \multirow[b]{2}{*}{ Mean } & \multirow{2}{*}{$\begin{array}{c}\text { Std. } \\
\text { deviation }\end{array}$} & \multirow{2}{*}{$\begin{array}{l}\text { Std. error } \\
\text { mean }\end{array}$} & \multicolumn{2}{|c|}{$\begin{array}{l}\text { 95\% confidence interval of the } \\
\text { difference }\end{array}$} & & & \\
\hline & & & & & Lower & Upper & & & \\
\hline Pair 1 & $\begin{array}{l}\text { Awake } \mathrm{SpO}_{2}-\text { Visit } \\
1-\text { Awake } \mathrm{SpO}_{2}- \\
\text { Visit } 2\end{array}$ & -2.054 & 4.037 & 0.469 & -2.989 & -1.119 & -4.377 & 73 & $<0.001$ \\
\hline Pair 2 & $\begin{array}{l}\text { Nadir } \mathrm{SpO}_{2}-\text { Visit } 1- \\
\text { nadir } \mathrm{SpO}_{2}-\text { Visit } 2\end{array}$ & -13.304 & 13.325 & 1.499 & -16.288 & -10.319 & -8.874 & 78 & $<0.001$ \\
\hline Pair 3 & $\begin{array}{l}\text { REM SpO } 2-\text { Visit } 1- \\
\text { REM SpO } 2 \text {-Visit } 2\end{array}$ & -2.422 & 7.467 & 1.113 & -4.665 & -0.179 & -2.176 & 44 & 0.035 \\
\hline
\end{tabular}

\section{OrCID}

\section{Arup K Halder (1) https://orcid.org/0000-0002-2255-9505}

\section{References}

1. Franklin KA, Lindberg E. Obstructive sleep apnea is a common disorder in the population-a review on the epidemiology of sleep apnea. J Thorac Dis 2015;7(8):1311-1322. DOI: 10.3978/j.issn.20721439.2015.06.11.

2. Kushida CA, Littner MR, Morgenthaler T, et al. Practice parameters for the indications for polysomnography and related procedures: an update for 2005. Sleep 2005;28(4):499-521. DOI: 10.1093/sleep/28.4.499.

3. Ahmadi N, Shapiro GK, Chung SA, et al. Clinical diagnosis of sleep apnea based on single night of polysomnography vs. two nights of polysomnography. Sleep Breath 2009;13(3):221-226. DOI: 10.1007/ s11325-008-0234-2.

4. Yamashiro Y, Kryger MH. CPAP titration for sleep apnea using a splitnight protocol. Chest 1995;107(1):62-66. DOI: 10.1378/chest.107.1.62.

5. Elshaug AG, Moss JR, Southcott AM. Implementation of a splitnight protocol to improve efficiency in assessment and treatment of obstructive sleep apnoea. Intern Med J 2005;35(4):251-254. DOI: 10.1111/j.1445-5994.2005.00786.x.

6. Khawaja IS, Olson EJ, van der Walt C, et al. Diagnostic accuracy of split-night polysomnograms. J Clin Sleep Med 2010;6:357-362.

7. Kapur VK, Auckley DH, Chowdhuri S, et al. Clinical practice guideline for diagnostic testing for adult obstructive sleep apnea: an American Academy of Sleep Medicine clinical practice guideline. J Clin Sleep Med 2017;13(3):479-504. DOI: 10.5664/jcsm.6506.

8. Morgenthaler TI, Aurora RN, Brown T, et al. Practice parameters for the use of autotitrating continuous positive airway pressure devices for titrating pressures and treating adult patients with obstructive sleep apnea syndrome: an update for2007. An American Academy of Sleep Medicine report. Sleep 2008; 31(1):141. DOI: 10.1093/sleep/31.1.141.
9. Ip S, D'Ambrosio C, Patel K, et al. Auto-titrating versus fixed continuous positive airway pressure for the treatment of obstructive sleep apnea: a systematic review with meta-analyses. Syst Rev 2012;1:20. DOI: 10.1186/2046-4053-1-20.

10. Collop NA, Anderson WM, Boehlecke B, et al. Clinical guidelines for the use of unattended portable monitors in the diagnosis of obstructive sleep apnea in adult patients. Portable Monitoring Task Force of the American Academy of Sleep Medicine. J Clin Sleep Med 2007;3(7):737-747.

11. Collop NA, Tracy SL, Kapur V, et al. Obstructive sleep apnea devices for out-of-center (OOC) testing: technology evaluation. J Clin Sleep Med 2011;7(5):531-548. DOI: 10.5664/JCSM.1328.

12. Flemons WW, Littner MR, Rowley JA, et al. Home diagnosis of sleep apnea: a systematic review of the literature. An evidence review cosponsored by the American Academy of Sleep Medicine, the American College of Chest Physicians, and the American Thoracic Society. Chest 2003;124(4):1543-1579. DOI: 10.1378/ chest.124.4.1543.

13. Ferber R, Millman R, Coppola $M$, et al. Portable recording in the assessment of obstructive sleep apnea. ASDA standards of practice. Sleep 1994;17(4):378-392. DOI: 10.1093/sleep/17.4.378.

14. Chuang ML, Lin IF, Vintch JR, et al. Predicting continuous positive airway pressure from a modified split-night protocol in moderate to severe obstructive sleep apnea-hypopnea syndrome. Intern Med 2008;47(18):1585-1592. DOI: 10.2169/internalmedicine.47.1107.

15. Kushida CA, Chediak A, Berry RB, et al. Positive Airway Pressure Titration Task Force; American Academy of Sleep Medicine. Clinical guidelines for the manual titration of positive airway pressure in patients with obstructive sleep apnea. J Clin Sleep Med 2008;4(2): 157-171. PMID: 18468315; PMCID: PMC2335396.

16. Shaarawy H, Gharraf HS. Comparison between the use of APAP and manual titration during split night polysomnography for diagnosis and treatment of OSA. Egypt J Chest Dis Tuberc 2018;67(2):175-181. DOI: 10.4103/ejcdt.ejcdt_20_18. 